\title{
On the analysis of Rayleigh wave in elastic media
}

\author{
K. G. Bhatia (*) - J. C. DRakopoulos $\left(^{* *}\right)$
}

Received on October 20, 1971

Summarr. - In this paper the Rayleigh wave equation has been simplified to two simple equations, the bifurcation point being the ratio of shear to longitudinal wave velocity. The purpose of the study is to obtain the phase velocity without being involved into the solution of six degree equation when the constants of the media are known.

Riassunto. - In questa nota l'equazione delle onde di Rayleigh ̀̀ stata rilotta a due semplici equazioni, il cui punto di intersezione rappresenta il rapporto fra la velocità delle onde superficiali (trasversali) e (quella delle onde longitudinali. Scopo dello studio è di ottenere la velocitì di fase, quando lo costanti dei mezzi sono conosciute, evitando cosi di risolvere un'equazione di sesto grado.

\section{Ixtroduction}

It is shown in treatises on theory of elasticity that two types of wave motion are propagated in an isotropic homogeneous and unbounded elastic medium. These are the longitudinal or the compressional $(P)$ waves and the transverse or the shear $(S)$ waves. Their velocities are respectively given by $\sqrt{\frac{\lambda+2 \mu}{\varrho}}$ and $\sqrt{\frac{\mu}{\varrho}}$ where $\lambda, \mu$ are Lame's constants of the media and $\varrho$ is the density. In any media the $P$ waves travel faster than the $S$ waves. All the earthquake records show the presence of surface waves in addition to these.

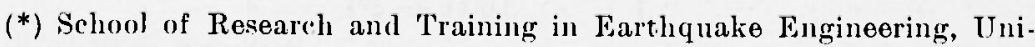
versity Roorkee, India.

(**) Seismological Laboratory, Athens University, Greece. 
The surface waves comprising the Rayleigh and Isove waves are distinguished from the bodily $P$ and $S$ waves in being more or less confined to the surface. Thus, their amplitudes decrease very rapidly with depth below the surface. It has always been the necessity to obtain the phase velocities of surface waves in order to analyse the behaviour of these waves in any media. In this paper the authors have paid particular attention on the Rayleigh type of wave only.

\section{Formelation of the probiem axi) (Conchesion}

The well known Rayleigh wave equation is $(1,2)$ :

$$
\xi^{2}\left[\xi^{6}-8 \xi^{4}+\xi^{2}\left(24-16 \eta^{2}\right)+16(\eta=-1)\right]=0
$$

where $\xi=\frac{c}{b}$ and $\eta=\frac{b}{a}, c$ is the phase velocity of Rayleigh wave in elastic media and $\eta$ is the ratio of shear wave velocity to compressional wave velocity. $a$ and $b$ are the longitudinal and shear wave

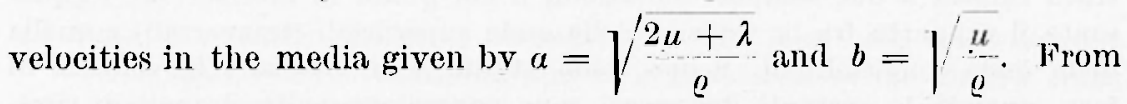
equation [1] we see that one root of $\xi$ is zero but this gives phase velocity equal to zero and hence is of not use.

Therefore from [1] we obtain

$$
\xi^{6}-8 \xi^{4}+\xi^{2}\left(24-16 \eta^{2}\right)+16\left(\eta^{2}-1\right)=0
$$

We may write it as

$$
\eta^{2}\left(16-16 \xi^{2}\right)+\xi^{6}-8 \xi^{4}+24 \xi^{2}-16=0
$$

or

$$
\eta^{2}=\frac{16-24 \xi^{2}+8 \xi^{4}-\xi_{6}}{16\left(1-\xi^{2}\right)}
$$

Therefore

$$
\eta=\sqrt{\left.\frac{16-24 \xi^{2}+8 \xi^{4}-\xi^{6}}{16\left(1-\xi^{2}\right)}\right]}
$$

Since $c$ is always less than $b, \xi$ is always less than unity. 
From [4] we can obtain a plot of $\eta$ versus $\xi$.

We can also solve the equation [2] for $\xi$ for different values of $\eta$. A simple computer program was written for this and the results were obtained.

Iaximum value of $\eta$ has been taken as 0.70 because for $\eta$ higher than this, Poisson's ratio becomes negative and no material yet has been obtained with negative Poisson's ratio ${ }^{(3)}$.

$$
\begin{gathered}
\text { Table I } \\
\xi_{0}=0.95529
\end{gathered}
$$

\begin{tabular}{|c|c|c|}
\hline$y$ & 5 & $\xi \quad \xi$ \\
\cline { 2 - 3 } 0.00 & & \\
0.05 & 0.95529 & 0.00 \\
0.10 & 0.95520 & -0.00009 \\
0.15 & 0.95465 & -0.00064 \\
0.20 & 0.95393 & -0.00136 \\
0.25 & 0.95274 & -0.00255 \\
0.30 & 0.95111 & -0.00418 \\
0.35 & 0.94904 & -0.00625 \\
0.40 & 0.94634 & -0.00895 \\
0.45 & 0.94286 & -0.01243 \\
0.50 & 0.93835 & -0.01694 \\
0.55 & 0.93249 & -0.02280 \\
0.60 & 0.92473 & -0.03056 \\
0.65 & 0.91416 & -0.04113 \\
0.70 & 0.89936 & -0.05593 \\
& 0.87789 & -0.07740 \\
\hline
\end{tabular}

In order to make the first value equal to zero, $\xi_{o}=0.95529$ has been subtracted from each value. $A \log -\log$ plot is obtained for $\left(\xi-\xi_{0}\right) v_{\delta}$ $\eta$ (see fig. 1).

For $0 \leqslant \eta \leqslant 0.40$ we get one straight line and for values $0.40<\eta \leqslant 0.70$ we get another straight line. Each straight line will give an equation of the type $y=k x^{\text {sec }}$ i.e $\xi-\xi_{0}=l_{*} \eta^{\text {sec }}$. Exact results are obtained by adjusting the values of constant $k$.

From graph

for $0<\eta \leqslant 0.40$

$$
\xi=0.95529-(0.10+0.05 \eta) \eta^{2.275}
$$

and for $0.40<\eta \leqslant 0.70$

$$
\xi=0.95529-(0.12+0.10 \eta) \eta^{2.83}
$$


Therefore for $0<\eta \leqslant 0.4$

$$
c=0.95529 b-(0.10+0.5 \eta) b \eta^{2.275}
$$

and for $0.4<\eta \leqslant 0.7$

$$
c=0.95529 b-(0.12+0.10 \eta) b \eta^{2.83}
$$

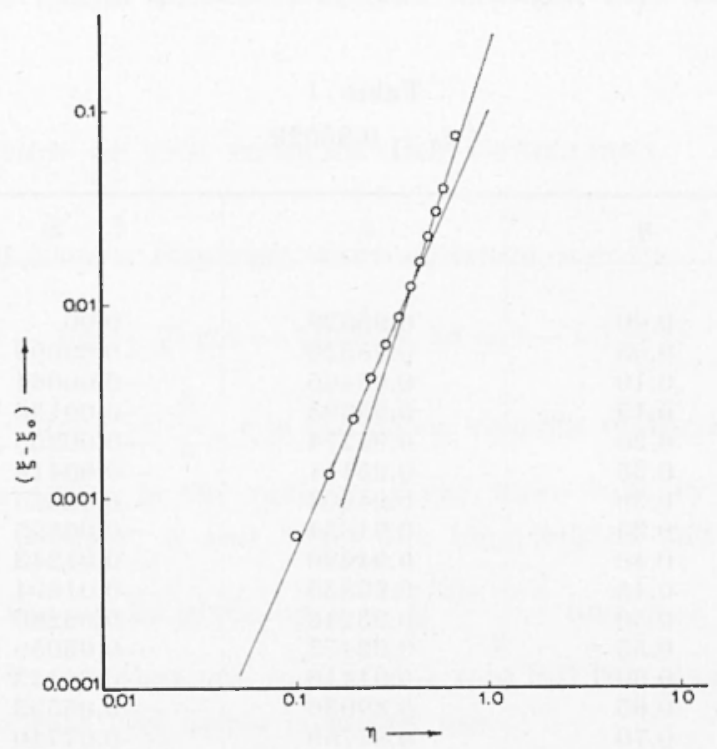

Fig. 1 - Reduced Rayleigh wave equation.

Jquations [5] and [6] or more practically [7] and [8] are reduced Rayleigh wave equation and these give results correct to third decimal place.

The values of $b=\sqrt{\frac{\mu}{\varrho}}$ and $\eta=\sqrt{\frac{\mu}{\lambda+2 \mu}}$ are calculated from the griven values and hence the phase velocity can instantly, be obtdined.

It can be noted that the phase velocity variation in both of the zones on $\eta$ i.e $0<\eta \leqslant 0.4$ and $0.4<\eta \leqslant 0.7$ is different.

It is expected that such equations will help in the analysis of Rayleigh wave under certain strict boundary conditions. Also, in order to analyse the Rayleigh wave in anelastic media, where the dissipation accompanies vibration, equations of these two type will help in obtaining the numerical solution of the problem. 


\section{REFERENCES}

(1) Bhatia K. G., and Inakopoulos J. C., 1971. - A study on the Displacement Components of Rayleigh wave. "Annali di Geofisica", XXIV, I, pl. 89-101.

(2) BUtLex K. E., 1963. - An Introduction to theory of Seismology. Camb. liniv. Press.

(3) Huaxg Y. T., 1968. - Effect of an Elastically Restrained Boundary on SV.Wave radialion pallerns. "Bull. Seism. Soc. Am.", pp. 497-520. 